\title{
Carga de enfermedad en años de vida ajustados por discapacidad del cáncer gástrico en Colombia
}

\author{
Disease Burden of Gastric Cancer in Disability-Adjusted Life Years in Colombia
}

Juan José Triana, ${ }^{1}$ Juan Diego Aristizábal-Mayor, ${ }^{1}$ María Camila Plata, ${ }^{1}$ Mauricio Medina, ${ }^{1}$ Laura Baquero, ${ }^{1}$ Sebastián Gil-Tamayo, ${ }^{1}$ Ana María Leguizamón, ${ }^{1}$ Felice Leonardi, ${ }^{2}$ Camilo Castañeda-Cardona, ${ }^{3}$ Diego Rosselli. ${ }^{4}$

Facultad de Medicina, Pontificia Universidad Javeriana, Bogotá, Colombia.

2 Eli Lilly Colombia, Bogotá, Colombia.

3 Neuroeconomix, Bogotá, Colombia.

4 Departamento de Epidemiología Clínica y

Bioestadística, Facultad de Medicina, Pontificia Universidad Javeriana, Bogotá, Colombia. Correo electrónico: diego.rosselli@gmail.com.

\begin{abstract}
Resumen
Objetivo: el cáncer gástrico es la segunda causa de muerte por cáncer y la quinta neoplasia más frecuente en el mundo. En Colombia, es la primera causa de mortalidad por cáncer. La incidencia y mortalidad anuales son 16,3 y 14,2/100 000 habitantes, respectivamente. El objetivo de este estudio fue estimar su carga de enfermedad, medida en años de vida ajustados por discapacidad (AVAD), en Colombia. Métodos: se desarrolló un estudio con enfoque en prevalencia para el año 2014. Para estimar la prevalencia, se realizó una búsqueda en los registros del Sistema de Información en Protección Social (SISPRO) y el Departamento Administrativo Nacional de Estadística (DANE). La duración promedio de los casos prevalentes y la sobrevida estimada se obtuvieron de la literatura local. Los AVAD fueron calculados sumando los años de vida perdidos por muerte prematura (AVPM) y los años de vida vividos con discapacidad (AVVD), según la metodología de la Organización Mundial de la Salud (OMS). Resultados: las prevalencias estimadas para 5 años en población mayor de 15 años fueron 40,9/100 000 en mujeres y 62,5/100 000 en hombres. El total de AVAD fue 293,418, con una tasa de 623/100 000 habitantes, de los cuales el 97,4\% corresponde a AVPM. La tasa de AVVD y AVPM para Colombia fue 16 y $607 / 100$ 000, respectivamente. Conclusiones: los datos obtenidos de SISPRO y el DANE estiman una alta carga de enfermedad en Colombia. Es necesaria la implementación de estrategias de detección temprana del cáncer para disminuir la carga de la enfermedad y mejorar el pronóstico de los pacientes.
\end{abstract}

\section{Palabras clave}

Neoplasias gástricas; costo de enfermedad; prevalencia; bases de datos factuales.

\begin{abstract}
Objective: Gastric cancer is the second most common cause of cancer death and the fifth most common neoplasm in the world. In Colombia, it is the leading cause of cancer mortality. The annual incidence and mortality are $16.3 / 100,000$ and 14.2/100,000 inhabitants respectively. The aim of this study was to estimate the disease burden in Colombia as measured in disability-adjusted life years (DALYs). Methods: This study focuses on prevalence in 2014. To estimate prevalence, a search was made in the registries of the Social Protection Information System (SISPRO) and the National Administrative Department of Statistics (DANE). The average duration of cases and estimated survival were obtained from the local literature. DALYs were calculated by adding the years of life lost due to premature death (YLLs) and years of life lived with disability (YLD) according to the methodology of the World Health Organization (WHO). Results: Prevalences estimated for five years in the population older than 15 years were $40.9 / 100,000$ for women and $62.5 / 100,000$ for men. The total DALY was 293,418 , with a rate of $623 / 100,000$ inhabitants; $97.4 \%$ correspond to YLL. The YLD and YLL for Colombia were 16/100,000 and 607/100,000, respectively. Conclusions: Data obtained from SISPRO and DANE estimate a high disease burden in Colombia. It is necessary to implement early cancer detection strategies to reduce the burden of disease and improve patient prognosis.
\end{abstract}

\section{Keywords}

Gastric neoplasms; cost of illness; prevalence; factual databases. 


\section{INTRODUCCIÓN}

El cáncer gástrico genera una carga global considerable, al ser la segunda causa de muerte por cáncer en el mundo después del cáncer de pulmón (1) y la quinta neoplasia más frecuente a nivel mundial para ambos sexos (2).

La epidemiología de la enfermedad varía considerablemente por región y por sexo, debido a la diferencia en hábitos alimenticios, edad y otros factores de riesgo de la población (3). Las regiones con mayor incidencia son Asia y Europa del Este, seguidas por Suramérica; en Norteamérica y el sur de África la incidencia es menor (3). La tasa de supervivencia en general es $<20 \%$ a 5 años (4). El diagnóstico tardío del cáncer gástrico se debe en gran parte a la presencia de síntomas iniciales inespecíficos, que llevan a un diagnóstico en estados avanzados. Sin embargo, en Japón y Corea se evidencia un aumento en la supervivencia, que llega a 90\% debido al diagnóstico temprano y a la resección endoscópica de la lesión $(1,4)$.

En Colombia, el cáncer gástrico es la primera causa de mortalidad de todos los cánceres. Tiene una incidencia anual de 16,3/100 000 habitantes y una mortalidad calculada de 14,2/100 000 habitantes (5). De acuerdo con la literatura local, el $41 \%$ de los pacientes en el momento del diagnóstico están en estadio III, y $21 \%$ en estadio IV, según los criterios de la American Joint Committee on Cancer (AJCC) (6). Según un estudio observacional en 1039 pacientes con cáncer gástrico, la supervivencia es menor que la reportada a nivel mundial; se calcula una tasa de supervivencia a 5 años de $11 \%$ (7).

La carga de la enfermedad atribuible a cáncer es de 7,8\% del total de AVAD a nivel mundial, en el que el cáncer gástrico aporta 0,7\% del total de AVAD $(8,9)$. En Brasil, donde se realizó un estudio de carga de la enfermedad de cáncer, se estimó que del total de AVAD generados por el cáncer, el 95,4\% correspondió AVPM (10). Esta cifra es considerablemente más alta que la registrada en países desarrollados, donde los AVPM son alrededor del $80 \%$ para cáncer, en general. Esto muestra la relevancia de carga de enfermedad del cáncer gástrico y la necesidad de realizar un diagnóstico temprano junto con el desarrollo de programas de detección temprana de la enfermedad (10).

El objetivo de este estudio fue estimar la carga, medida en AVAD, del cáncer gástrico en Colombia, utilizando los datos locales de prevalencia, y empleando la metodología establecida por la OMS (11-13).

\section{MATERIALES Y MÉTODOS}

El estudio se realiza sobre datos de prevalencia del cáncer gástrico en Colombia para el año 2014. Para estimar la prevalencia de cáncer gástrico en Colombia, se realizó una búsqueda en los registros del SISPRO. La fuente principal de SISPRO es el Registro Individual de Prestación de Servicios de Salud (RIPS), centralizador de todos los datos relacionados con atención individual en salud en Colombia, labor a cargo del Ministerio de Salud y Protección Social.

Para realizar el análisis se tomaron los datos de 5 años de los RIPS, de 2010 al 2014. Se incluyeron los códigos de la clasificación internacional de enfermedades -CIE10- relacionados con cáncer gástrico desde C160 a C166, incluyendo C169 (tumor maligno del cardias, fondo gástrico, cuerpo del estómago, antro pilórico, píloro, curvatura menor y curvatura mayor del estómago, y tumor maligno del estómago, parte no especificada). Estos diagnósticos fueron escrutados con 2 filtros de diagnóstico: confirmado nuevo y confirmado repetido; excluyendo el tipo de diagnóstico, impresión diagnóstica y no especificado.

Con el fin de cuantificar el número de pacientes con este diagnóstico, se utilizó la función "personas atendidas", que incluye solo una vez a la persona, así haya sido atendida más de una vez durante los 5 años. Después de obtener estos datos, se discriminaron por sexo, grupo etario (por quinquenios) y localización geográfica por departamento.

Para la estimación de la prevalencia de cáncer gástrico en el año 2014, se utilizó como denominador poblacional la información del DANE, agrupando la población por quinquenios, estimada para el año en mención. Los datos del DANE están basados en el censo poblacional realizado en 2005 en Colombia (www.dane.gov.co) y sus respectivas proyecciones, aunque hay evidencia de que puede estar subestimando la población de mayor edad $(14,15)$. Se estimó la prevalencia por sexo y por grupo etario de igual forma que la prevalencia general, utilizando como denominador el estimado poblacional según el DANE para estos subgrupos.

\section{ESTIMACIÓN DE LOS AVAD}

Los AVAD se calcularon a partir de la suma de los AVPM (en inglés, years of life lost o YLL) y los AVVD (en inglés, years lived with disability o YLD) de acuerdo con la metodología de la OMS propuesta desde el año 2013 (11, 12). De esta manera, al usar los AVPM se considera la mortalidad atribuible a una enfermedad, mientras que con los AVVD se mide la morbilidad de la misma. Se aplicó una expectativa de vida al nacer de 86 años para hombres y mujeres, y para cada grupo etario se empleó la expectativa de vida para ese rango de edad (por ejemplo, para el grupo de edad de 75 a 79 años, la expectativa es de 12,9 años, y para los de 80 y más es de 9,3 años), sin tasa de descuento ni ajuste por edades (11). Aunque otros estudios de carga de la enfermedad emplean la expectativa de vida real de cada país (16), la OMS recomienda estandarizar esta población para así permitir comparaciones internacionales. 
Aunque la nueva metodología abre el debate acerca del empleo de incidencia o prevalencia para el cálculo de los AVAD, los investigadores hemos considerado establecer un enfoque desde la prevalencia, con el interés de permitir el cálculo de AVVD en la población sobreviviente con desenlaces crónicos. Los pesos de discapacidad (en una escala entre $0 \mathrm{y}$ 1 , donde 0 es perfecta salud) para los estados de salud secundarios a cáncer gástrico se basaron en los estudios de Salomon y colaboradores y Murray y colaboradores $(8,11)$. El cambio metodológico de emplear prevalencia y no incidencia genera un aumento en los AVAD totales para todas las causas (12).

La estimación de los AVVD se realizó a partir de la prevalencia estimada y una ponderación de los diferentes pesos de discapacidad para cáncer gástrico de acuerdo con la metodología de Murray. La proporción de pacientes que hipotéticamente se ubicarían en cada uno de los 4 estados de salud (desde oligosintomático hasta terminal) se obtuvo a partir de un estudio realizado del 2004 al 2008 en Colombia (6) y de la asesoría del panel de expertos gastroenterólogos con experiencia en el manejo de pacientes con cáncer gástrico. Los AVVD fueron categorizados según grupos de edad de la siguiente manera: 0-4 años, 5-14 años, 15-29 años, 30-44 años, 45-59 años, 60-69 años, 70-79 años y 80 años o más. $\mathrm{Al}$ ser un estudio con un enfoque de prevalencia, no se incluyó la duración promedio de la enfermedad en el cálculo de los AVVD.

Los AVPM se calcularon a partir de la diferencia entre la edad de muerte del paciente y la expectativa de vida planteada por la OMS (86 años para hombres y mujeres), teniendo como base la prevalencia obtenida del RIPS y del DANE. No se utilizó tasa de descuento alguna para los AVPM. La información se organizó en los mismos rangos de edad.

\section{ANÁLISIS DE DATOS}

Todos los datos obtenidos de las distintas fuentes (RIPS, DANE y literatura) se organizaron en hojas de cálculo en Excel para su análisis y el cálculo de prevalencia en 5 años para cáncer gástrico, supervivencia, duración promedio en años de los pacientes con cáncer gástrico y tasa de letalidad anual. Posteriormente, con la plantilla de la OMS para el cálculo de carga de la enfermedad, se obtuvieron los AVAD (13).

\section{RESULTADOS}

Los datos consultados de los RIPS en los años 2010 al 2014 mostraron que en Colombia se atendieron 15972 pacientes (9467 hombres, 59,3\%; y 6495 mujeres, $40,7 \%$ ) con diagnóstico confirmado de cáncer gástrico (Tabla 1). De acuerdo con estos datos, las prevalencias estimadas para 5 años en población mayor de 15 años son 40,9/100 000 en mujeres y 62,5/100 000 en hombres (Tabla 2).
Los quinquenios a partir de los 55 años hasta los 74 años mostraron un aumento significativo en el número de pacientes, cuyo registro superó los 2000 pacientes atendidos. El número de atenciones informadas para pacientes durante los años 2010 a 2014 fue de 96 805; comparando el número de atenciones con el número de pacientes en total atendidos, se puede estimar que, en promedio, cada paciente recibió 6 atenciones.

El total de muertes obtenido, de acuerdo con la tasa de mortalidad, fue de 10 797; estos datos se discriminaron según quinquenios y se reagruparon en estos 8 grupos etarios: 0-4 años, 5-14 años, 15-29 años, 30-44 años, 45-59 años, 60-69 años, 70-79 años y 80 años o más. Del total de muertes, 3057 correspondieron al año 2014. A partir de estos datos se calcularon los AVPM totales $(81069)$ y los AVPM por 100000 habitantes $(170,1)$.

El total de AVVD se calculó a partir de los casos determinados para cada grupo etario, así como a partir del promedio de supervivencia de la enfermedad reportado en la literatura nacional (21 meses) (7). Para el cálculo de AVVD se tiene en cuenta el reporte de 4 "estados de salud" para el cáncer gástrico: diagnóstico y terapia primaria (peso de discapacidad de 0,294), etapa metastásica (peso de discapacidad de 0,484 ), etapa terminal (peso de discapacidad de $0,508)$ y fase controlada (peso de discapacidad de 0,031 ) $(8,11)$. En conjunto con el análisis del panel de expertos y lo descrito en la literatura, se estableció en qué porcentaje del tiempo promedio de supervivencia permanecerían, de forma hipotética, los pacientes en cada estado de salud. Aplicando $15 \%$ para diagnóstico y terapia primaria, 30\% para etapa metastásica, $15 \%$ para etapa terminal y $40 \%$ para fase controlada, se calculó un peso de discapacidad ponderado de 0,278 para el tiempo vivido con cáncer gástrico.

El total de AVAD fue de 82326 , con una tasa de 172,7/100 000 habitantes; el 98,5\% corresponde a AVPM (Tabla 3). La tasa de AVVD para la población colombiana fue 2,6/100 000 habitantes. El grupo etario con más AVAD fue el de 45-59 años, que aporta 38,7\% del total, seguido del grupo de 60-69 años.

\section{DISCUSIÓN}

A pesar de que el cáncer gástrico es el primer tipo de cáncer en mortalidad en Colombia (2), ha recibido menos atención que otras formas de cáncer como el cervicouterino, el de mama o el de próstata. En términos de incidencia, es el primero en hombres, seguido del cáncer de próstata; mientras que en mujeres ocupa el cuarto lugar después de cáncer de mama, cuello uterino y colorrectal (2). De acuerdo con estas cifras, que mostraron una incidencia ajustada por edad de 26,5 y $15,4 / 100000$ personas-año en hombres y mujeres, respectivamente (17); según datos del Instituto 
Tabla 1. Personas atendidas con diagnóstico principal de cáncer gástrico por grupos de edad durante el período 2010-2014. Datos RIPS

\begin{tabular}{|c|c|c|c|c|c|c|}
\hline Edad & 2010 & 2011 & 2012 & 2013 & 2014 & Total general* \\
\hline 0 a 4 años & 3 & & & 1 & 7 & 10 \\
\hline 5 a 9 años & 3 & & & 3 & 1 & 7 \\
\hline 10 a 14 años & 2 & & 1 & 2 & 2 & 5 \\
\hline 15 a 19 años & 42 & 9 & 8 & 11 & 28 & 93 \\
\hline 20 a 24 años & 19 & 11 & 22 & 22 & 28 & 95 \\
\hline 25 a 29 años & 41 & 27 & 32 & 41 & 53 & 180 \\
\hline 30 a 34 años & 72 & 68 & 79 & 86 & 106 & 329 \\
\hline 35 a 39 años & 105 & 130 & 144 & 133 & 178 & 546 \\
\hline 40 a 44 años & 205 & 242 & 262 & 241 & 279 & 943 \\
\hline 45 a 49 años & 273 & 344 & 385 & 366 & 431 & 1363 \\
\hline 50 a 54 años & 344 & 409 & 505 & 496 & 567 & 1756 \\
\hline 55 a 59 años & 379 & 467 & 604 & 550 & 675 & 2039 \\
\hline 60 a 64 años & 395 & 566 & 598 & 583 & 723 & 2171 \\
\hline 65 a 69 años & 391 & 486 & 592 & 540 & 727 & 2183 \\
\hline 70 a 74 años & 427 & 541 & 597 & 539 & 694 & 2214 \\
\hline 75 a 79 años & 296 & 405 & 475 & 434 & 579 & 1769 \\
\hline 80 años o más & 296 & 437 & 502 & 474 & 606 & 1925 \\
\hline Total general & 3180 & 4048 & 4664 & 4412 & 5471 & 15972 \\
\hline
\end{tabular}

*La columna Total general muestra el número total de personas que se atendieron en algún momento del período, no necesariamente corresponde a la suma de atendidos por año.

Tabla 2. Prevalencia a 5 años (entre 2010 y 2014) de cáncer gástrico en hombres y mujeres, usando diagnóstico principal, expresado en quinquenios

\begin{tabular}{lccccccccc}
\hline & \multicolumn{3}{c}{ Mujer } & \multicolumn{3}{c}{ Hombre } & \multicolumn{3}{c}{ Total general $^{*}$} \\
\hline \multicolumn{1}{c}{ Quinquenios } & Población & Casos & Prevalencia & Población & Casos & Prevalencia & Población & Casos $^{\text {Prevalencia }}$ \\
\hline 0-4 años & 2104983 & 3 & 0,1 & 2205140 & 7 & 0,3 & 4310123 & 11 & 0,3 \\
5-9 años & 2082905 & 4 & 0,2 & 2178087 & 3 & 0,1 & 4260992 & 7 & 0,2 \\
10-14 años & 2104802 & 4 & 0,2 & 2196672 & 1 & 0,0 & 4301474 & 7 & 0,2 \\
15-19 años & 2135740 & 49 & 2,3 & 2231913 & 44 & 2,0 & 4367653 & 98 & 2,2 \\
20-24 años & 2081630 & 51 & 2,5 & 2182627 & 43 & 2,0 & 4264257 & 102 & 2,4 \\
25-29 años & 1927690 & 93 & 4,8 & 1961986 & 87 & 4,4 & 3889676 & 194 & 5,0 \\
30-34 años & 1778575 & 174 & 9,8 & 1700271 & 153 & 9,0 & 3478846 & 411 & 11,8 \\
35-39 años & 1616660 & 294 & 18,2 & 1525194 & 252 & 16,5 & 3141854 & 690 & 22,0 \\
40-44 años & 1491010 & 465 & 31,2 & 1378539 & 478 & 34,7 & 2869549 & 1229 & 42,8 \\
45-49 años & 1505806 & 579 & 38,5 & 1373306 & 783 & 57,0 & 2879512 & 1799 & 62,5 \\
50-54 años & 1371402 & 739 & 53,9 & 1245714 & 1017 & 81,6 & 2617116 & 2321 & 88,7 \\
55-59 años & 1126190 & 751 & 66,7 & 1008294 & 1286 & 127,5 & 2134484 & 2675 & 125,3 \\
60-64 años & 876569 & 783 & 89,3 & 782667 & 1387 & 177,2 & 1659236 & 2865 & 172,7 \\
65-69 años & 666944 & 797 & 119,5 & 583881 & 1385 & 237,2 & 1250825 & 2736 & 218,7 \\
70-74 años & 485493 & 852 & 175,5 & 406288 & 1360 & 334,7 & 891781 & 2798 & 313,8 \\
75-79 años & 381431 & 697 & 182,7 & 293335 & 1072 & 365,5 & 674766 & 2189 & 324,4 \\
80 años o más & 392287 & 847 & 215,9 & 277356 & 1076 & 387,9 & 669643 & 2315 & 345,7 \\
Total & 24130117 & 6495 & 26,9 & 23531670 & 9467 & 40,2 & 47661789 & 15972 & 35,5 \\
\hline
\end{tabular}

*La columna Total general no corresponde a la sumatoria sino al número de personas atendidas en algún momento del quinquenio. La prevalencia se calcula con la población media del período como denominador. 
Tabla 3. Distribución de AVAD, AVPM y AVVD por cáncer gástrico en Colombia, según quinquenios, para el año 2013 . Tasas por 100000 habitantes

\begin{tabular}{lccccc}
\hline \multicolumn{1}{r}{ Quinquenio } & Población & AVAD & Tasa AVAD & Tasa AVPM & Tasa AVVD \\
\hline $0-4$ & 4310122 & 57 & 1,3 & 1,3 & 0,0 \\
$5-14$ & 8562463 & 261 & 3,0 & 3,0 & 0,0 \\
$15-29$ & 12521536 & 3084 & 24,6 & 24,5 & 0,2 \\
$30-44$ & 9489938 & 14805 & 156,0 & 154,7 & 1,3 \\
$45-59$ & 7630157 & 32842 & 430,4 & 425,2 & 5,1 \\
$60-69$ & 2909302 & 17970 & 617,7 & 606,8 & 10,7 \\
$70-79$ & 1565889 & 10192 & 650,9 & 633,3 & 17,3 \\
80 o más & 669323 & 3116 & 465,6 & 445,7 & 19,7 \\
Total & 47658730 & 82326 & 172,7 & 170,1 & 2,6 \\
\hline
\end{tabular}

Nacional de Cancerología, Colombia se considera como un país de alta incidencia y mortalidad por cáncer gástrico a nivel mundial.

Los datos obtenidos a partir del SISPRO son de origen oficial, recolectados por el Ministerio de Salud y Protección Social de Colombia. No obstante, el registro de RIPS está sujeto a un adecuado diligenciamiento del diagnóstico, según la CIE-10, que realicen los médicos en las historias clínicas. Es sensato asumir que algunos de estos registros no se realizan de manera óptima, hecho que genera errores en la base de datos, más probablemente por subregistro. Estudios previos de nuestro grupo sugirieron que la precisión diagnóstica de los RIPS mejora para aquellas enfermedades que, como la esclerosis múltiple (18), el diagnóstico está a cargo de un grupo seleccionado de especialistas y cuyo tratamiento requiere destinar recursos importantes por parte del sistema de salud.

Incluso teniendo en cuenta el posible subregistro en los RIPS, en este estudio se encontró una prevalencia más alta de cáncer gástrico de la prevista por la iniciativa Globocan en 2012 para Colombia (20,6 para mujeres y 37,5 para hombres) y similar a la entregada por la International Agency for Research in Cancer (IARC) de la OMS, que estima la prevalencia e incidencia para Colombia a partir de datos de un sistema de vigilancia que cubre menos del $10 \%$ de la población $(2,14,19)$. Aunque estos datos son los de mejor calidad a nivel nacional, solamente provienen de registros regionales tomados de 4 ciudades (Cali, Pasto, Manizales y Bucaramanga) (20-23).

Es relevante anotar que, en un análisis preliminar de las cifras reportadas por el DANE sobre defunciones ocasionadas directamente por cáncer gástrico, hubo 23253 muertes por esta enfermedad a nivel nacional en el período 2010-2014, lo que supera el número total de casos reportados en los RIPS (24). Estas cifras oficiales son de valor significativo como referencia objetiva del número de pacientes con cáncer gástrico al momento del fallecimiento y refuerzan la necesidad de mejorar los sistemas de vigilancia de cáncer a nivel nacional.
Para el cálculo de los AVAD en Colombia, se tomaron las proyecciones del DANE para 2014, basadas en el censo de 2005, dado que son las de más alta confiabilidad a nivel nacional. La gran mayoría de AVAD calculados fueron aporte de los AVPM debido a la letalidad de esta enfermedad, para la cual se calcula una supervivencia a 5 años de apenas 1 de cada 9 pacientes (7). Al comparar los resultados de este estudio con estudios regionales en Chile, país con un comportamiento epidemiológico similar al colombiano, se evidencia una supervivencia de 9,6\% a 5 años (25) (comparable con el $11 \%$ encontrado en el estudio de Vries, en Bucaramanga) (7). En países en vías de desarrollo, los AVAD totales dependen en más de un 90\% de los AVPM. En cambio, en países desarrollados, la proporción se acerca al 80\% (10).

La carga de la enfermedad en Colombia para cáncer gástrico reportada en la literatura es menor a la de otros países con incidencias similares. Aunque Colombia es uno de los países con mayor incidencia de cáncer gástrico del mundo, la tasa reportada de AVAD (304/100 000 habitantes) (9) es similar a la de países con incidencia intermedia. Países con incidencias elevadas y programas estrictos de tamización y curación temprana del cáncer gástrico, como Corea del Sur y Japón, donde la proporción de AVPM en los AVAD es menor, cuentan con tasas muy superiores a las colombianas, donde el diagnóstico es tardío y hay más muertes prematuras en edades productivas al afectar a adultos jóvenes (26). Aunque los datos obtenidos en nuestro estudio a partir de la base de datos del SISPRO y la mortalidad del DANE estiman una carga de enfermedad más elevada, todavía pueden estar subestimando la verdadera magnitud del problema (27).

En conclusión, Colombia cuenta con una incidencia importante de cáncer gástrico que indica la necesidad de trabajar más sobre aspectos como el registro adecuado ya que, como lo muestra este estudio, se reportan más defunciones que número de consultas por paciente en el sistema de salud asociadas con esta enfermedad. Otros aspectos relevantes derivados de la evaluación de los datos del estudio, en los que se debe trabajar con más énfasis, son: 
tamizaje, diagnóstico temprano, seguimiento periódico de evolución de la enfermedad y tratamiento oportuno, con el fin de disminuir la carga de la enfermedad y así mejorar el pronóstico de los pacientes.

\section{Conflicto de interés}

Este trabajo recibió el apoyo económico de Eli Lilly Interamérica, Colombia.

\section{REFERENCIAS}

1. Correa P. Gastric cancer: overview. Gastroenterol Clin North Am. 2013;42(2):211-7. https://doi.org/10.1016/j. gtc.2013.01.002

2. Ferlay J, Soerjomataram I, Dikshit R, et al. Cancer incidence and mortality worldwide: sources, methods and major patterns in GLOBOCAN 2012. Int J Cancer. 2015;136(5):E359-86.

3. Thrumurthy SG, Chaudry MA, Hochhauser D, et al. The diagnosis and management of gastric cancer. BMJ. 2013;347:f6367.

4. Quiros RM, Bui CL. Multidisciplinary approach to esophageal and gastric cancer. Surg Clin North Am. 2009;89(1):79-96. https://doi.org/10.1016/j.suc.2008.09.019

5. Pardo C, Cendales R. Incidencia, mortalidad y prevalencia de Cáncer en Colombia 2007-2011. 1. a edición. Bogotá D.C: Instituto Nacional de Cancerología; 2015.

6. Oliveros R, Navarrera LF. Diagnosis, staging and treatment of gastric cancer in Colombia from 2004 to 2008. Rev Colomb Gastroenterol. 2012;27(4):269-74.

7. de Vries E, Uribe C, Pardo C, et al. Gastric cancer survival and affiliation to health insurance in a middle-income setting. Cancer Epidemiol. 2015;39(1):91-6. https://doi. org/10.1016/j.canep.2014.10.012

8. Murray CJ, Ezzati M, Flaxman AD, et al. GBD 2010: design, definitions, and metrics. Lancet. 2012;380(9859):2063-6. https://doi.org/10.1016/S0140-6736(12)61899-6

9. Institute for Health Metrics and Evaluation. GBD compare. IHME [internet] 2016 [acceso marzo 23 de 2017]. Disponible en: http://vizhub.healthdata.org/gbd-compare.

10. Traebert J, Schneider IJ, Colussi CF, et al. Burden of disease due to cancer in a Southern Brazilian state. Cancer Epidemiol. 2013;37(6):788-92. https://doi.org/10.1016/j. canep.2013.08.007

11. Salomon JA, Vos T, Hogan DR, et al. Common values in assessing health outcomes from disease and injury: disability weights measurement study for the Global Burden of Disease Study 2010. Lancet. 2012;380(9859):2129-43. https://doi.org/10.1016/S0140-6736(12)61680-8

12. Mathers C, Stevens G. WHO methods and data sources for global burden of disease estimates 2000-2011. WHO [internet] 2013 [acceso marzo 23 de 2017]. Disponible en: http:// cdrwww.who.int/ healthinfo/ statistics/ GlobalDALYmethods_2000_2011.pdf.
13. WHO. Health statistics and information systems. WHO [internet] 2017 [acceso marzo 23 de 2017]. Disponible en: http://www.who.int/healthinfo/global_burden_disease/ tools_national/en/

14. Rosselli D, Hernández-Galvis J. El impacto del envejecimiento sobre el sistema de salud colombiano. Salud Publica Mex. 2016;58(6):595-596. https://doi.org/10.21149/spm. v58i6.7880

15. Henríquez Mendoza G, de Vries E. El efecto del envejecimiento parala carga de cánceren Colombia: proyeccionesparalas primeras cinco localizaciones por departamento y sexo en Colombia, 2020 y 2050. Rev Colomb Cancerol. 2017;21(2):104-12. https://doi.org/10.1016/j.rccan.2017.04.002

16. De Vries E, Meneses MX, Piñeros M. Years of life lost as a measure of cancer burden in Colombia, 1997-2012. Biomedica. 2016;36(4):547-55. https://doi.org/10.7705/ biomedica.v36i4.3207

17. Pardo C, Cendales R. Incidencia estimada y mortalidad por cáncer en Colombia, 2002-2006. Bogotá: Instituto Nacional de Cancerología; 2010.

18. Jiménez-Pérez CE, Zarco-Montero LA, Castañeda-Cardona $\mathrm{C}$, et al. Estado actual de la esclerosis múltiple en Colombia. Acta Neurol Colomb 2015;31(4):385-90.

19. Allemani C, Weir HK, Carreira H, et al. Global surveillance of cancer survival 1995-2009: analysis of individual data for $25,676,887$ patients from 279 populationbased registries in 67 countries (CONCORD-2). Lancet. 2015;385(9972):977-1010. https://doi.org/10.1016/ S0140-6736(14)62038-9

20. Uribe C, Osma S, Herrera V. Cancer incidence and mortality in the Bucaramanga metropolitan area, 2003-2007. Colomb Med (Cali). 2012;43(4):290-7.

21. Yépez MC, Bravo LE, HidalgoTroya A, et al. Cancer incidence and mortality in the municipality of Pasto, 1998 2007. Colomb Med (Cali). 2012;43(4):256-66.

22. López-Guarnizo GA, Arias-Ortiz NE, Arboleda-Ruiz WA. Cancer incidence and mortality in Manizales 2003-2007. Colomb Med (Cali). 2012;43(4):281-9.

23. Bravo LE, Collazos T, Collazos P, et al. Trends of cancer incidence and mortality in Cali, Colombia. 50 years experience. Colomb Med (Cali). 2012;43(4):246-55.

24. Departamento Administrativo Nacional de Estadística. Defunciones no fetales. DANE [internet] 2015 [acceso marzo 23 de 2017]. Disponible en: http://www.dane.gov. co/index.php/estadisticas-por-tema/salud/nacimientos$\mathrm{y}$-defunciones/defunciones-no-fetales/defunciones-nofetales-2015.

25. Heise K, Bertran E, Andia ME, et al. Incidence and survival of stomach cancer in a high-risk population of Chile. World J Gastroenterol. 2009;15(15):1854-62. https://doi. org/10.3748/wjg.15.1854

26. Machii R, Saika K. Estimated Disability-Adjusted Life Year (DALY) in Asia in GLOBOCAN 2008. Jpn J Clin Oncol. 2013;43(8):846-7. https://doi.org/10.1093/jjco/hyt107

27. Alba LH, Alba M, Ortiz D, et al. Análisis de los registros individuales de prestación de servicios de salud (RIPS) en cáncer en Colombia. Medicina (Bogotá). 2016;38(3):223-31. 